\title{
Commissioning of the LHCb Particle ID and Tracking systems
}

\section{David Hutchcroft*}

University of Liverpool, UK

E-mail: David.Hutchcroftecern.ch

The proposed calibration with data and expected performance of the LHCb particle identification and tracking systems is discussed.

12th International Conference on B-Physics at Hadron Machines

September 7-11, 2009

Heidelberg, Germany.

* Speaker. 


\section{Introduction}

The LHCb detector is a purpose designed $b$ physics experiment at the Large Hadron Collider [1]. The detector is designed with an acceptance in the forward region of the proton-proton collisions and will have $10^{12} \mathrm{~b} \bar{b}$ pairs in the acceptance in a year of data taking at nominal luminosity. The expected performance of the detector and how to calibrate it using data will be discussed.

\section{2. $\mathrm{LHCb}$ detector}

The LHCb detector is described in Ref. [2]. LHCb comprises of several subsystems covering a nominal angular acceptance of $250 \mathrm{mrad}$ (vertical) and $300 \mathrm{mrad}$ (vertical):

VELO is 88 half disks of silicon surrounding the interaction region sensitive between 8 and $42 \mathrm{~mm}$ in radius, split into two retractable halves on either side of the interaction region [3]

TT is two pairs of planes of silicon strip detectors at a distance of about $2.5 \mathrm{~m}$ from the nominal interaction point (IP), with the strips vertical in two planes and at a $5^{\circ}$ stereo in the other two [4]

OT is three stations of $0.5 \mathrm{~cm}$ diameter straw tubes with 4 double layers of straws and a $5^{\circ}$ stereo in half of the planes between 8 and $9.5 \mathrm{~m}$ from the IP [5]

IT is a cross shaped arrangement of 2 pairs of silicon strip planes around the beam pipe, covering the region that would have more than $10 \%$ occupancy in the OT at high luminosity [4]

RICH1 is a ring imaging Čerenkov detector using both an aerogel and $\mathrm{C}_{4} \mathrm{~F}_{10}$ gas radiator to determine particle types, from 100 to $200 \mathrm{~cm}$ from the IP [6]

RICH2 is a second RICH detector for higher momentum particles at about $10 \mathrm{~m}$ from the IP using a $\mathrm{CF}_{4}$ gas radiator, but only covers the first $120 \mathrm{mrad}$ acceptance in the horizontal plane [6]

CALO is a calorimeter system with four distinct planes of detectors to provide sensitivity for electromagnetic and hadronic showers and the ability to separate electrons from photons for the trigger [7]

MUON is 5 layers of chambers, one in front of the calorimeter, separated by $80 \mathrm{~cm}$ walls of iron to identify charged particles as muons [8]

\section{Tracking}

The tracking performance is driven by the VELO for the track position at the production position and by the overall bend measured in OT and IT after the magnetic field for the momentum estimate. The efficiency to find tracks crossing the whole detector with a momentum higher than $10 \mathrm{GeV} / \mathrm{c}$ is about $94 \%$, with a rate of fake (ghost) tracks of $9 \%$ [2]. Almost all of the ghost tracks can be removed by a cut on the minimum transverse momentum of the tracks.

The nominal VELO performance should result in the impact parameter resolution shown in Fig. 1. The performance can be degraded by unknown detector misalignment and magnetic field 

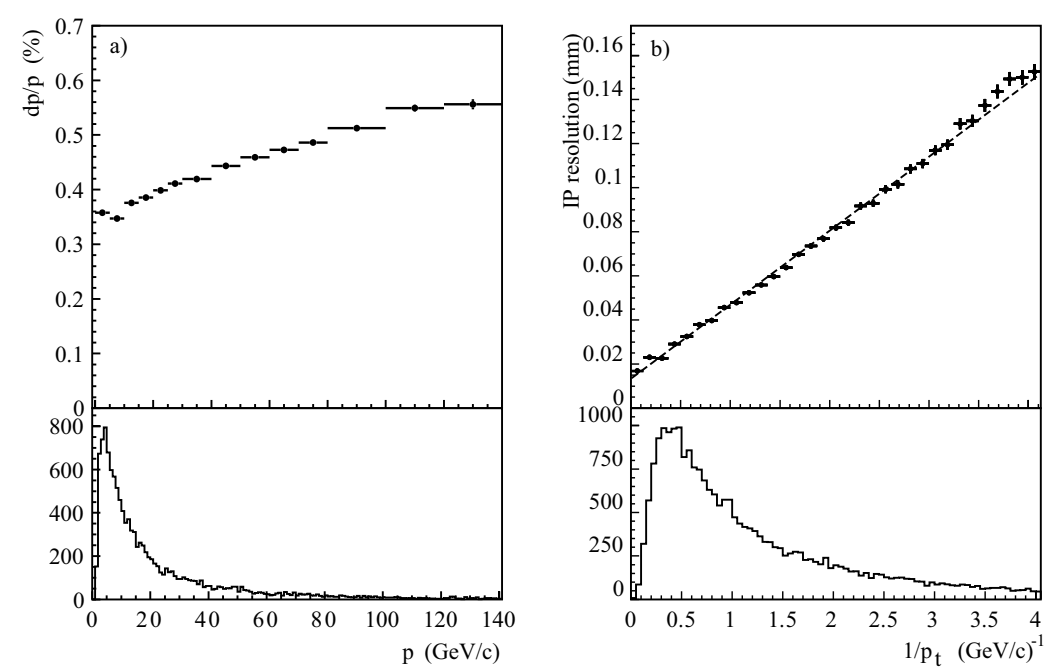

Figure 1: The momentum resolution (a) and the impact parameter resolution (b) for the tracks reconstructed crossing the whole tracking system of the LHCb detector [2]. Below are the expected parameter distributions for $b$ daughter tracks in each variable.

uncertainties. After construction each VELO half was placed in a coordinate measuring machine and surveyed, providing a measurement of the relative detector positions to better than $10 \mu \mathrm{m}$ [9]. During operations the detector positions will be monitored and further refined by looking at the residuals from each reconstructed track in each sensor. The magnetic field was surveyed by a robot with many Hall probes, and the three components of the magnetic field are known to $0.4 \%$ precision, for both the field up and field down configurations [10]. The main source of the uncertainty is the variation in the measurements of the same point by different Hall probes after they were all calibrated. The variation in the magnetic field from this map will be monitored by reconstructing $K_{s}^{0} \rightarrow \pi^{+} \pi^{-}$and $\Lambda^{0} \rightarrow p \pi^{-}$decays. The lifetimes of these particles are such that there is significant separation from the primary vertex before their decays in flight and almost all backgrounds can be rejected. About three quarters of the reconstructible $K_{s}^{0}$ and $\Lambda^{0}$ decay after the VELO and variations in the reconstructed masses verse reconstructed $K_{s}^{0} / \Lambda^{0}$ vertex position and subsequent decay particle trajectories will show where the magnetic field map needs adjustment. The crosssection for $\Lambda^{0}$ production is about $15 \mathrm{mb}$ so there will be enough reconstructed $\Lambda^{0}$ decays in all events recorded without requiring a dedicated trigger, the $K_{s}^{0}$ decays are even more copious.

\section{Particle identification}

The particle identification performance for pions, kaons and protons is predominately done by the two RICH detectors. To provide the samples of pions and kaons the decay chain $B_{d} \rightarrow D^{*+} \rightarrow$ $D^{0}\left(K^{-} \pi^{+}\right) \pi^{+}$is reconstructed, where a cut on the mass difference between $D^{*+}$ and $D^{0}$ leaves the a clean sample of $D^{0}$ decays without requiring particle identification on the daughter tracks. The charge of the slow pion identifies the type of the other two tracks. The kaons and pions from the $D^{0}$ are then used to calibrate the ring size and number of photons per track observed in each RICH detector. The $\Lambda^{0}$ decays provide a clean set of both protons and pions. 

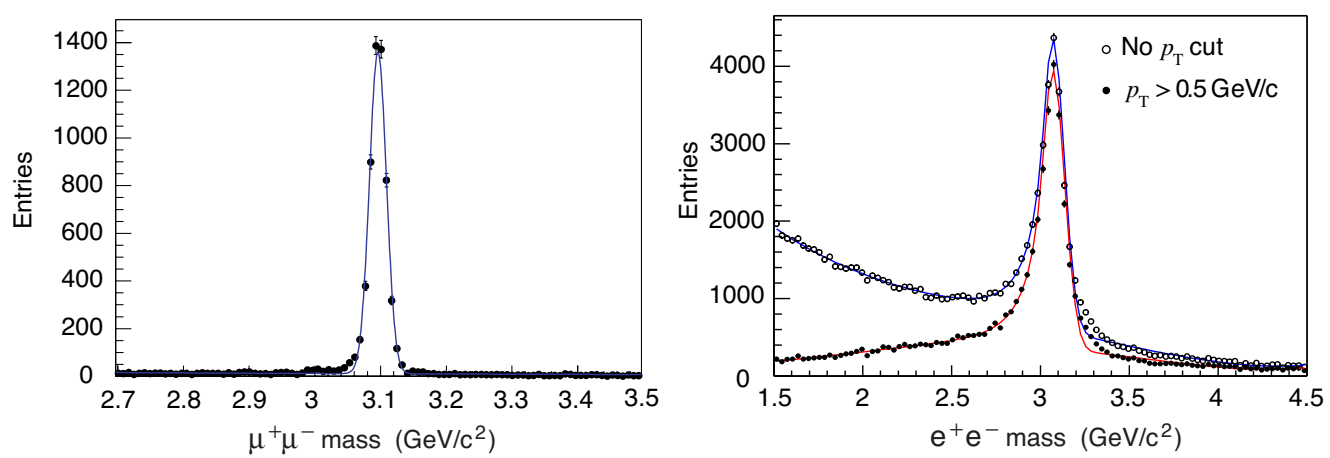

Figure 2: The reconstructed $J / \psi$ mass peaks for $\mu^{+} \mu^{-}$(left) and $e^{+} e^{-}$(right) decays for signal MC events [2]. In the case of the electron decay channel an additional cut on the $p_{T}$ of the electron tracks is applied to remove ghost tracks from the decays.

The identification of muons is predominately done by the Muon system, with muons identified by hits in a window around the extrapolated track direction. The window sizes will be tuned on data using samples of muons from $J / \psi \rightarrow \mu^{+} \mu^{-}$decays for signal, see Fig. 2. While $\Lambda^{0} \rightarrow p \pi^{-}$ will provide backgrounds of both pions, which can decay in flight, and protons which do not. The $J / \psi \rightarrow \mu^{+} \mu^{-}$has a cross-section of about $290 \mu$ b, a trigger selection has been developed that can select about $240 \mathrm{~Hz}$ of $J / \psi \rightarrow \mu^{+} \mu^{-}$events using only the tracking system and one layer of the muon detectors on one of the muons. This can be pre-scaled once the required number of unbiased muons have been collected.

In LHCb electron reconstruction has two phases, first identifying tracks as electrons and then adding the energy of any bremsstrahlung photons to the electrons track momentum. Bremsstrahlung only happens in material, so none is caused as the trajectory crosses the magnet volume, as the magnet has no material other than air and the beam pipe inside it. The bremsstrahlung photons that are produced will be in the material in front of the magnet or the tracking stations behind it, where the trajectories are nearly straight. These photons can be recovered by extrapolating the tracks from the VELO and from the OT and IT stations to the calorimeters and adding the energy of the photons found to that of the electron's calorimeter cluster. The electron identification will be calibrated with $J / \psi \rightarrow e^{+} e^{-}$events using the fully reconstructed B decay and one tagged electron. The bremsstrahlung recovery will be tuned by optimising the reconstructed $J / \psi$ mass peak.

$\pi^{0}$ reconstruction is difficult at a hadron collider, there are substantial photon and other neutral backgrounds. $\mathrm{LHCb}$ will be the first experiment at a hadron collider to reconstruct a $b$ decay with neutrals in the final state. The $\pi^{0}$ can be reconstructed as resolved or merged depending on whether both photons are reconstructed separately in the calorimeter or only one electromagnetic cluster is seen. In LHCb resolved $\pi^{0}$ are more common below $4 \mathrm{GeV}$ and merged above that energy, at $4 \mathrm{GeV}$ the $\pi^{0}$ efficiency is about $50 \%$ [2]. The scale of the electromagnetic clusters will be calibrated by the electrons in the $J / \psi \rightarrow e^{+} e^{-}$channel, see Fig. 2, and the mass of the reconstructed $\pi^{0}$. There will also be a significant fraction of photons that convert to $e^{+} e^{-}$, they will be reconstructed separately and provide an additional set of photons and electrons to reconstruct the $\pi^{0}$ mass peak, see Fig. 3. 

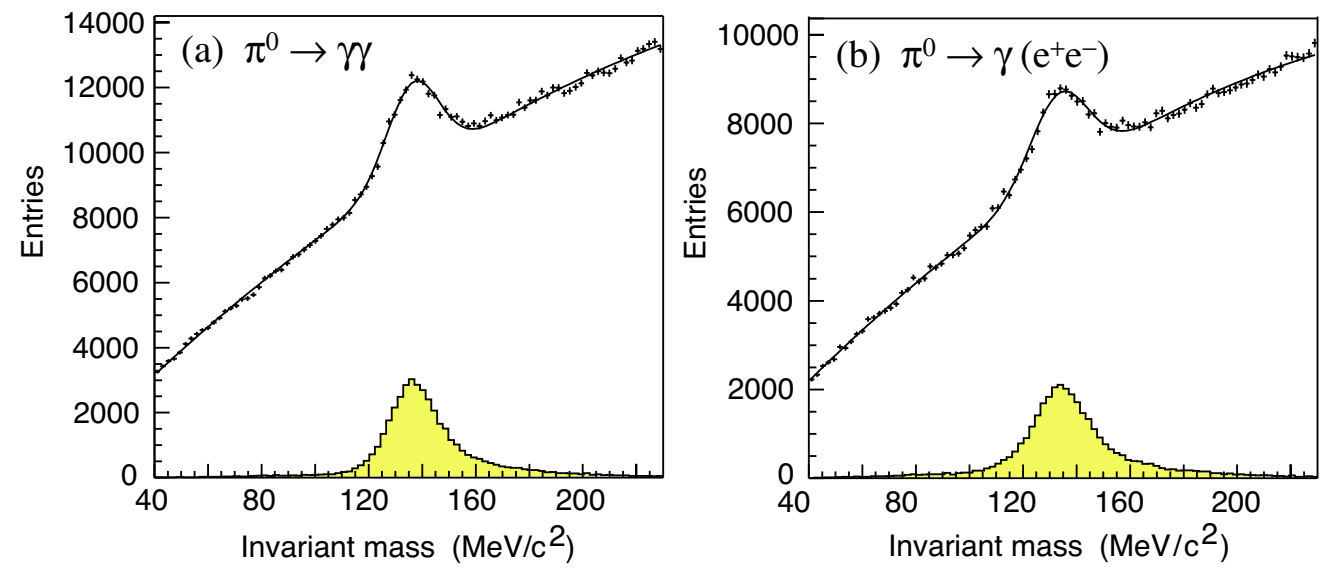

Figure 3: $\pi^{0}$ mass distributions, where (a) neither photon has converted, (b) one $\gamma$ converted before the calorimeter [2]. The contributions of true $\pi^{0}$ are indicated by the shaded histograms.

When reconstructing $\mathrm{B}$ decays the lifetime measurement and the invariant mass resolution are two of the most important parameters to discriminate from backgrounds and to perform time dependant $\mathrm{CP}$ violation measurements. The time resolution is around $40 \mathrm{fs}$ fitted with a single Gaussian for $B_{s} \rightarrow D_{s}^{ \pm} K^{\mp}$ decays, fitted with a double Gaussian gives a core resolution of $33 \pm 1 \mathrm{fs}$ and a tail containing $31 \%$ of the events with a resolution of $67 \pm 3 \mathrm{fs}$.

The mass resolutions of the reconstructed B masses will be $14 \mathrm{MeV} / \mathrm{c}^{2}$ for $B_{s} \rightarrow D_{s}^{ \pm} K^{\mp}$, $15 \mathrm{MeV} / \mathrm{c}^{2}$ for $B_{s} \rightarrow J / \psi(\mu \mu) \phi, 17 \mathrm{MeV} / \mathrm{c}^{2}$ for $B \rightarrow h^{+} h^{-}$(where $h$ is $\pi$ or $K$ ) and $64 \mathrm{MeV} / \mathrm{c}^{2}$ for $B_{d} \rightarrow K^{*} \gamma$. The resolution varies with the number of charged particles in the decay and is relatively poor when a neutral is reconstructed as part of the decay.

\section{Conclusion}

The $\mathrm{LHCb}$ detector is expected to provide the largest sample of reconstructed B decays of any experiment to date. Well calibrated tracking and particle identification are necessary to achieve the best physics results from the very large data set the experiment will collect. As presented in this article all of the groups responsible for a component of the detector has made detailed measurements during construction and have a strategy to calibrate and understand the particle reconstruction using the very first data taken by the experiment.

\section{References}

[1] L. Evans and P. Bryant (ed.), "LHC Machine," 2008 JINST 3 S08001

[2] The LHCb Collaboration, "The LHCb Detector at the LHC," 2008 JINST 3 S08005

[3] LHCb Collaboration, "LHCb Vertex Locator TDR", CERN-LHCC-2001-028

[4] LHCb Collaboration, "LHCb Inner Tracker TDR”, CERN-LHCC-2002-029

[5] LHCb Collaboration, "LHCb Outer Tracker TDR”, CERN-LHCC-2001-041 
[6] LHCb Collaboration, "LHCb RICH TDR”, CERN-LHCC-2000-050

[7] LHCb Collaboration, "LHCb Calorimeters TDR", CERN-LHCC-2000-049

[8] LHCb Collaboration, “LHCb Muon System TDR”, CERN-LHCC-2001-029

[9] LHCb VELO group, "Report on VELO-alignment campaign of October 2007", CERN EDMS note 908162, https://edms.cern.ch/document/908162/1

[10] M. Losasso et al., "Tests and Field Map of LHCb Dipole Magnet", IEEE Transactions on Applied Superconductivity, Volume 16, Issue 2, pages 1700-1703, June 2006. 